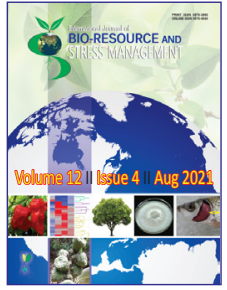

\title{
Crop Regulation in Pomegranate (Punica granatum L.) through Induced Water Stress and Ethrel Application
}

\author{
R. Kumar*, P. L. Saroj and B. D. Sharma
}

ICAR-Central Institute for Arid Horticulture, Bikaner, Rajasthan (334 006), India

Open Access

Corresponding Author

R. Kumar

e-mail: rameshflori@gmail.com

Citation: Kumar et al., 2021. Crop Regulation in Pomegranate (Punica granatum L.) through Induced Water Stress and Ethrel Application. International Journal of Bio-resource and Stress Management 2021, 12(4), 309-318. HTTPS://DOI ORG/10.23910/1.2021.2378.

Copyright: (c) 2021 Kumar et al. This is an open access article that permits unrestricted use, distribution and reproduction in any medium after the author(s) and source are credited.

Data Availability Statement: Legal restrictions are imposed on the public sharing of raw data. However, authors have full right to transfer or share the data in raw form upon request subject to either meeting the conditions of the original consents and the original research study. Further, access of data needs to meet whether the user complies with the ethical and legal obligations as data controllers to allow for secondary use of the data outside of the original study.

Conflict of interests: The authors have declared that no conflict of interest exists.

Acknowledgements: The authors are grateful to the ICAR-Central Institute for Arid Horticulture, Bikaner for providing required facilities and guidance to conduct the research.

\begin{abstract}
The present investigation was carried out at ICAR-CIAH, Bikaner, Rajasthan, India during 2017 to 2019 to regulate crop production in pomegranate at the desired period with higher number of bisexual flower for higher quality fruit yield and to minimize fruit splitting through water stress, pruning and ethrel application. Pooled data analysis revealed that horticultural interventions, chemical treatments and their interactions significantly improved plant volume, bisexual flower number, maturity index, fruit yield with significant reduction in fruit splitting, mite and fungal spot incidence. Among different treatment combinations, maximum canopy volume, number of bisexual flowers, maturity index, fruit yield and lower fruit splitting were recorded $\left(8.56 \mathrm{~m}^{3}, 85.11\right.$ plant $^{-1}, 47.81,124.01 \mathrm{q} \mathrm{ha}^{-1}$ and $\left.1.87 \mathrm{~kg} \mathrm{plant}^{-1}\right)$ in withholding irrigation during June+pruning+thinning+ethrel $2 \mathrm{ml} \mathrm{l}^{-1}$ as compared to absolute control $\left(3.41 \mathrm{~m}^{3}, 75.62\right.$ plant $^{-1}, 33.35,85.66$ $\mathrm{q} \mathrm{ha} \mathrm{a}^{-1}$ and $2.41 \mathrm{~kg}$ plant $\left.{ }^{-1}\right)$, respectively. The mite and fungal spot incidence were recorded minimum (4.72 and $8.50 \%$ ) in withholding irrigation during June+pruning+thinning+ethrel $3 \mathrm{ml} \mathrm{l}^{-1}$ application in comparison to maximum recorded in absolute control ( 29.73 and $33.68 \%)$, respectively. Therefore, it can be concluded that withholding irrigation during June+pruning+thinning with ethrel $2 \mathrm{mll}^{-1}$ treatment significantly improved quality fruit yield with higher number of bisexual flowers and reduced fruit splitting through induction of flowering and fruit growth during optimal climatic conditions.
\end{abstract}

Keywords: Bisexual flower, ethrel, fruit splitting, pomegranate, water stress

\section{Introduction}

Pomegranate (Punica granatum L.) is a promising fruit crop of hot arid and semi-arid regions of India. The fruit is mainly used for table, juice purposes and also processed in various value-added products like syrup, squash, jelly, mouth freshener and anardana. Pomegranate has enormous medicinal and nutritional value and it is one of the richest sources of antioxidants particularly anthocyanins. Owing to health promoting substances, it is considered as "Super food or food medicine". In India, it is grown commercially in Maharashtra, Karnataka, Andhra Pradesh, Gujarat, Telangana, Tamil Nadu, Madhya Pradesh and Rajasthan states. The area under pomegranate cultivation in India is increasing at faster rate owing to its high demand, hardy nature, better storage quality and nutritional values (Saroj and Kumar, 2019). Due to its hardy nature,

Article History

RECEIVED on $21^{\text {st }}$ May 2021 RECEIVED in revised form on $08^{\text {th }}$ August 2021 ACCEPTED in final form on $19^{\text {th }}$ August 2021 
high yield and low input requirements it has emerged as a potential fruit crop for the resource poor farmers under harsh conditions (Rajkumar et al., 2017). It covers 273 thousand ha area with an annual production of 3068 thousand $t$ and a productivity of $11.24 \mathrm{t} \mathrm{ha}^{-1}$ in India (Anonymous, 2021). Pomegranate fruit production requires the coordination of numerous key reproductive processes including flower development, pollination, bisexual and male flower types, fruit set, fruit attributes related to size, flower vigour, flower receptivity and aril development (Wetzstein et al., 2015). In pomegranate male flowers drop prematurely without setting fruit and only bisexual flowers set fruits (Yahya et al., 2017). Pomegranate plant flowers in three main seasons or bahars during January-February (ambe bahar), June-July (mrig bahar) and September-October (hasta bahar). Only one season flowering and fruiting is regulated to get prolific harvest at a specific time. In areas where assured rainfall occurs during June-September, flowering in June-July is advantageous, where monsoon starts late in August, flowering during August is beneficial. Areas where irrigation water available during April-May, flowering during January-February is suggested and where monsoon withdraws by September, induction of flowering in October is possible (Sachin et al., 2015). In hot arid region, mrig bahar crop is preferred but fruits are severely damaged by fruit splitting during December-January owing to day night temperature fluctuations. Owing to inferior colour development and quality of fruits, both hasta and ambe bahar are not recommended in the arid climate (Singh and Kingsly, 2007). Water stresses causes a wide array of plant responses, ranging from plant growth, leaf defoliation, flower induction and finally productivity (Kumar et al., 2019a). In pomegranate fruit development, water stress causes an asymmetric increase in fruit turgor pressure, because aril turgor increased to a much greater extent than peel turgor, the pressure of the arils on the peel favouring fruit splitting (Galindo et al., 2014), which is a major problem in hot arid climate. In pomegranate crop regulation, water stress, plant growth regulators, defoliants, nutrient and plant architectural engineering are major horticultural innovations which influence flowering induction, alter sex ratio and improve fruit quality (Kumar et al., 2019b). Crop regulation in pomegranate is done keeping in mind the availability of irrigation, pest and disease infestation and market prices (Shivran et al., 2020). In pomegranate, 1-2 month stress is imposed by the withholding irrigation depending upon soil and climatic condition. Pruning and flower/bud thinning is performed to modify natural growth habits of pomegranate plant and to make balance between vegetative and reproductive growth which regulate flowering and fruiting for quality yield. Withholding of irrigation during June+pruning and thinning+ethrel $2 \mathrm{ml}$ $\mathrm{l}^{-1}+$ DAP $5 \mathrm{~g} \mathrm{l}^{-1}$ treatment induced flowering during optimal climatic conditions and significantly improved sex ratio and fruit set of pomegranate (Kumar et al., 2020).

Ethylene cause an array of effects in plants such as breaking of dormancy, leaf abscission, senescence and induce, promote and delay flowering, alter sex expression, defoliate plants, flower and fruits thinning and enhance colour development (Abeles et al., 1992). When the plants are subjected to ethylene, gene expression of cell wall-degrading enzymes particularly cellulase and polygalacturonase are activated. Ethylene is engaged in the arrest of stamen development by the initiation of DNA damage, which promotes female flowers in some plant species (Wang et al., 2010 and Xie et al., 2015). Application of ethylene $0.2 \mathrm{ml} \mathrm{l}^{-1}$ reduced bacterial blight incidence, enhanced fruit yield/quality; increased phenol and anthocyanin content in pomegranate (Lalithya et al., 2017). The translocation of sugars and water to the arils tends to be the cause of the rise in juice volume (Supe and Saitwal, 2016). The maturity index is an important criterion for fruit quality determination which is often better related to palatability of fruit than either sugar or acid level alone. It varies from 31.90 to 37.90 among different cultivars (Dhinesh et al., 2017). Therefore, the present investigation was carried out to regulate crop production in desired period in such a way that plant produces higher number of bisexual flower with improved quality fruit yield with reduction in fruit splitting, mite and fungal spot incidence by forcing plants to go into rest through withholding irrigation, pruning and ethrel application.

\section{Materials and Methods}

The present investigation was conducted at Research Field of ICAR-Central Institute for Arid Horticulture, Bikaner during two consecutive years during 2017 to 2019. There were two factors i.e. horticultural interventions $(P)$ with five levels and chemical applications (C) with four levels. The horticultural interventions involved $P_{0}$ control natural flowering, $P_{1}$ withholding irrigation during March, $\mathrm{P}_{2}$ withholding irrigation during March+pruning+thinning, $\mathrm{P}_{3}$ withholding irrigation during June and $\mathrm{P}_{4}$ withholding irrigation during June+pruning+thinning whereas chemical applications consisted of $\mathrm{C}_{0}$ control without chemicals, $\mathrm{C}_{1}$ ethrel $1 \mathrm{ml}^{-1}, \mathrm{C}_{2}$ ethrel $2 \mathrm{ml} \mathrm{l}^{-1}$ and $C_{3}$ ethrel $3 \mathrm{ml} \mathrm{l}^{-1}$ comprising 20 treatment combinations. The experiment was conducted in Factorial Randomized Block Design with three replications. After withholding irrigation, pruning of $20 \mathrm{~cm}$ growth was carried out. In thinning, flowers were removed manually before and during water stress period as per the treatments and only flowers which induced after application of treatments were retained. Ethrel (40\%) mixed with $5 \mathrm{~g} \mathrm{l}^{-1}$ DAP solution (18:46 grade) was applied as foliar spray after withholding irrigation period and pruning as per the treatments, while in natural flowering treatments (control treatment of the first factor) ethrel mixed with $5 \mathrm{~g} \mathrm{l}^{-1}$ DAP was applied in last week of May. The treatments were imposed on eight years old uniform plants of pomegranate cv. Jalore Seedless planted at $5 \times 2.5$ $\mathrm{m}^{2}$ spacing with drip irrigation system. Uniform intercultural operations were performed to grow the crop. Recommended dose of manure and fertilizers i.e. 45 kg FYM, 625 g N, 250 
g $P$ and 250 g K plant $^{-1}$ year $^{-1}$ were applied. The orchard soil was loamy sand in nature with soil pH of 8.32 and electrical conductivity of $0.27 \mathrm{dSm}^{-1}$. The soil had $0.15 \%$ organic carbon, $106.4 \mathrm{~kg} \mathrm{ha}^{-1}$ available nitrogen, $11.51 \mathrm{~kg} \mathrm{ha}^{-1}$ available phosphorus and $214.5 \mathrm{~kg} \mathrm{ha}^{-1}$ available potassium content. The maximum temperature during April-May was recorded 42 to $44^{\circ} \mathrm{C}$ and minimum 4 to $5^{\circ} \mathrm{C}$ during December-January. The diurnal temperature variation was varied from minimum $10^{\circ} \mathrm{C}$ in July-August to maximum $20^{\circ} \mathrm{C}$ in November-December, which causes severe cracking in pomegranate under hot arid climatic conditions.

Data were recorded on plant height and volume, annual growth extension (AGE), number of male, intermediate and bisexual flowers per plant, flowering and harvesting period, aril and rind colour, fruit volume and juice content, maturity index, fruit splitting, fruit yield, mite and fungal spot incidence. The plant volume was worked out with the help of formula given by Westwood (1978) while aril and rind colour of the fruit were observed by comparing it with the colour chart of the Royal Horticultural Society, London. The total soluble solids of the fruit juice were determined with digital refractometer Atago PAL II and titrable acidity of juice was determined by titration with $0.1 \mathrm{~N}$ sodium hydroxide (Ranganna, 1995). The maturity index was determined as the ratio of total soluble solids and titrable acidity. The experimental data recorded were subjected to statistical analysis using the analysis of variance technique suggested by Panse and Sukhatme (1985).

\section{Results and Discussion}

The perusal of data presented in Table 1 revealed that plant height, volume and AGE were significantly affected by horticultural interventions, chemical applications and their interactions. The maximum plant height $(2.34 \mathrm{~m})$ was registered in withholding irrigation during June+pruning and thinning $\left(P_{4}\right)$ treatment which was statistically at par with withholding irrigation during June $\left(P_{3}\right)$ treatment $(2.30 \mathrm{~m})$. The minimum plant height $(2.14 \mathrm{~m})$ was registered in control $\left(P_{0}\right)$ treatment. The maximum plant volume $\left(6.24 \mathrm{~m}^{3}\right)$ was recorded in withholding irrigation during June+pruning and thinning $\left(\mathrm{P}_{4}\right)$ treatment which was followed by withholding irrigation during June $\left(P_{3}\right)$ treatment $\left(5.79 \mathrm{~m}^{3}\right)$. The minimum plant volume $\left(4.62 \mathrm{~m}^{3}\right)$ was registered in control $\left(P_{0}\right)$ treatment. Among chemical applications, significantly maximum plant height $(2.42 \mathrm{~m})$ and plant volume $\left(6.67 \mathrm{~m}^{3}\right)$ were recorded in ethrel $2 \mathrm{mll}^{-1}\left(\mathrm{C}_{2}\right)$ treatment as compared to minimum plant height $(2.09 \mathrm{~m})$ and plant volume $\left(4.37 \mathrm{~m}^{3}\right)$ recorded in control $\left(\mathrm{C}_{0}\right)$. Among interaction treatments, maximum plant height $(2.62$ $\mathrm{m})$ and plant volume $\left(8.56 \mathrm{~m}^{3}\right)$ were recorded in withholding irrigation during June+pruning and thinning+ethrel $2 \mathrm{ml} \mathrm{l}^{-1}$ $\left(\mathrm{P}_{4} \mathrm{C}_{2}\right)$ treatment which was followed by withholding irrigation during June+ethrel $2 \mathrm{mll}^{-1}\left(\mathrm{P}_{3} \mathrm{C}_{2}\right)$ treatment with plant height $(2.49 \mathrm{~m})$ and plant volume $\left(7.12 \mathrm{~m}^{3}\right)$ while minimum plant height $(1.94 \mathrm{~m})$ and plant volume $\left(3.41 \mathrm{~m}^{3}\right)$ were recorded in absolute control i.e. natural flowering without chemicals $\left(\mathrm{P}_{0} \mathrm{C}_{0}\right)$ treatment.
Table 1: Effect of crop regulation treatments on vegetative attributes of pomegranate

\begin{tabular}{|c|c|c|c|}
\hline Treatments & $\begin{array}{c}\text { Plant height } \\
\text { (m) }\end{array}$ & $\begin{array}{c}\text { Canopy } \\
\text { volume }\left(\mathrm{m}^{3}\right)\end{array}$ & $\begin{array}{l}\text { AGE } \\
(\mathrm{cm})\end{array}$ \\
\hline \multicolumn{4}{|c|}{ Horticultural interventions (P) } \\
\hline$P_{0}$ & 2.14 & 4.62 & 48.64 \\
\hline$P_{1}$ & 2.19 & 4.95 & 51.88 \\
\hline$P_{2}$ & 2.21 & 5.27 & 53.57 \\
\hline$P_{3}$ & 2.30 & 5.79 & 55.64 \\
\hline$P_{4}$ & 2.34 & 6.24 & 59.96 \\
\hline SEm \pm & 0.02 & 0.07 & 0.16 \\
\hline $\operatorname{CD}(p=0.05)$ & 0.06 & 0.21 & 0.48 \\
\hline \multicolumn{4}{|c|}{ Chemical applications (C) } \\
\hline $\mathrm{C}_{0}$ & 2.09 & 4.37 & 51.73 \\
\hline $\mathrm{C}_{1}$ & 2.25 & 5.42 & 54.62 \\
\hline$C_{2}$ & 2.42 & 6.67 & 56.00 \\
\hline $\mathrm{C}_{3}$ & 2.17 & 5.04 & 53.4 \\
\hline SEm \pm & 0.02 & 0.07 & 0.15 \\
\hline $\mathrm{CD}(p=0.05)$ & 0.05 & 0.19 & 0.44 \\
\hline \multicolumn{4}{|c|}{ Horticultural interventions $\times$ Chemical applications $(\mathrm{P} \times \mathrm{C})$} \\
\hline $\mathrm{P}_{0} \mathrm{C}_{0}$ & 1.94 & 3.41 & 46.74 \\
\hline $\mathrm{P}_{0} \mathrm{C}_{1}$ & 2.15 & 4.65 & 48.93 \\
\hline $\mathrm{P}_{0} \mathrm{C}_{2}$ & 2.36 & 6.02 & 50.46 \\
\hline $\mathrm{P}_{0} \mathrm{C}_{3}$ & 2.10 & 4.41 & 48.45 \\
\hline $\mathrm{P}_{1} \mathrm{C}_{0}$ & 2.11 & 4.33 & 50.42 \\
\hline $\mathrm{P}_{1} \mathrm{C}_{1}$ & 2.18 & 4.87 & 52.10 \\
\hline $\mathrm{P}_{1} \mathrm{C}_{2}$ & 2.35 & 5.97 & 54.12 \\
\hline $\mathrm{P}_{1} \mathrm{C}_{3}$ & 2.14 & 4.64 & 50.89 \\
\hline $\mathrm{P}_{2} \mathrm{C}_{0}$ & 2.13 & 4.75 & 51.75 \\
\hline $\mathrm{P}_{2} \mathrm{C}_{1}$ & 2.22 & 5.41 & 54.19 \\
\hline $\mathrm{P}_{2} \mathrm{C}_{2}$ & 2.32 & 5.71 & 55.48 \\
\hline $\mathrm{P}_{2} \mathrm{C}_{3}$ & 2.19 & 5.22 & 52.87 \\
\hline $\mathrm{P}_{3} \mathrm{C}_{0}$ & 2.13 & 4.53 & 53.54 \\
\hline$P_{3} C_{1}$ & 2.35 & 6.04 & 56.49 \\
\hline $\mathrm{P}_{3} \mathrm{C}_{2}$ & 2.49 & 7.12 & 57.43 \\
\hline $\mathrm{P}_{3} \mathrm{C}_{3}$ & 2.22 & 5.48 & 55.11 \\
\hline $\mathrm{P}_{4} \mathrm{C}_{0}$ & 2.15 & 4.85 & 56.22 \\
\hline $\mathrm{P}_{4} \mathrm{C}_{1}$ & 2.37 & 6.12 & 61.41 \\
\hline $\mathrm{P}_{4} \mathrm{C}_{2}$ & 2.62 & 8.56 & 62.53 \\
\hline $\mathrm{P}_{4} \mathrm{C}_{3}$ & 2.21 & 5.45 & 59.70 \\
\hline SEm \pm & 0.04 & 0.15 & 0.33 \\
\hline $\operatorname{CD}(p=0.05)$ & 0.12 & 0.42 & 0.98 \\
\hline
\end{tabular}


The data divulged that AGE was significantly affected by horticultural interventions, chemical applications and their interactions. Among horticultural interventions, significantly maximum AGE $(59.96 \mathrm{~cm})$ was recorded in withholding irrigation during June+pruning and thinning $\left(\mathrm{P}_{4}\right)$ treatment followed by withholding irrigation during June $\left(\mathrm{P}_{3}\right)$ treatment $(55.64 \mathrm{~cm})$ as against minimum AGE $(48.64 \mathrm{~cm})$ was recorded in control natural flowering $\left(P_{0}\right)$ treatment. Among chemical applications, maximum AGE $(56.00 \mathrm{~cm})$ was registered in ethrel $2 \mathrm{ml} \mathrm{l}^{-1}\left(C_{2}\right)$ treatment followed by ethrel $1 \mathrm{ml} \mathrm{l}^{-1}\left(C_{1}\right)$ treatment $(54.62 \mathrm{~cm})$ whereas minimum recorded in control without chemicals $\left(C_{0}\right)$ treatment $(51.73 \mathrm{~cm})$. Among interaction treatments, maximum AGE was recorded $(62.53 \mathrm{~cm})$ in withholding irrigation during June+pruning and thinning+ethrel $2 \mathrm{ml} \mathrm{l}^{-1}\left(\mathrm{P}_{4} \mathrm{C}_{2}\right)$ treatment in comparison to minimum recorded $(46.74 \mathrm{~cm})$ in absolute control $\left(P_{0} C_{0}\right)$ treatment. The enhanced plant height, volume and $A G E$ in withholding irrigation during June+pruning and thinning+ethrel $2 \mathrm{ml} \mathrm{l}^{-1}\left(\mathrm{P}_{4} \mathrm{C}_{2}\right)$ treatment could be attributed to the favourable climatic condition, low mite and fungal spot incidence which resulted in improved partitioning of assimilates. Pruning and thinning improved vegetative growth probably due to optimization of light penetration, ventilation and distribution of nutrients which improved photosynthetic rate. The ethrel at lower concentration might have acted as growth promoter by increasing cell division and enlargement and improved vegetative growth of plants. Amarnath et al. (2020) also recorded maximum plant height $(271.30 \mathrm{~cm})$ in $20 \mathrm{~cm}$ pruning as compared to minimum recorded in control without pruning $(214.57 \mathrm{~cm})$ in pomegranate.

The data revealed that (Table 2) number of male, intermediate and bisexual flowers per plant were significantly influenced by horticultural interventions, chemical application and their interactions. The significantly minimum number of male flowers plant ${ }^{-1}(165.95)$ was registered in withholding irrigation during June+pruning and thinning $\left(\mathrm{P}_{4}\right)$ treatment as compared to maximum male flowers plant ${ }^{-1}$ (213.49) recorded in control natural flowering $\left(\mathrm{P}_{0}\right)$ treatment. The significantly minimum number of intermediate flowers plant ${ }^{-1}$ (39.58) was recorded in withholding irrigation during June+pruning and thinning $\left(\mathrm{P}_{4}\right)$ treatment while maximum number of intermediate flowers plant ${ }^{-1}(47.42)$ was recorded in control natural flowering $\left(P_{0}\right)$ treatment. The significantly maximum number of bisexual flowers plant ${ }^{-1}(82.75)$ was observed in withholding irrigation during June+pruning and thinning $\left(\mathrm{P}_{4}\right)$ treatment while minimum number of bisexual flowers plant ${ }^{-1}$ (74.72) in withholding irrigation during March+pruning and thinning $\left(P_{2}\right)$ which was at par with control $\left(P_{0}\right)$ treatment (75.08). Among chemical treatments, minimum male flowers plant ${ }^{-1}(188.50)$ was registered in ethrel $2 \mathrm{mll}^{-1}\left(\mathrm{C}_{2}\right)$ treatment as compared to control without chemicals $\left(\mathrm{C}_{0}\right)$ treatment (192.32). The significantly minimum intermediate flowers plant $^{-1}$ (39.37) was registered in control without chemicals $\left(C_{0}\right)$ treatment as against maximum recorded in ethrel 2
Table 2: Effect of crop regulation treatments on male, intermediate and bisexual flowers of pomegranate

\begin{tabular}{|c|c|c|c|}
\hline Treatments & $\begin{array}{l}\text { Male flowers } \\
\left(\text { no. plant }{ }^{-1}\right)\end{array}$ & $\begin{array}{c}\text { Intermediate } \\
\text { flowers } \\
\text { (no. plant }{ }^{-1} \text { ) }\end{array}$ & $\begin{array}{c}\text { Bisexual } \\
\text { flowers } \\
\left(\text { no. plant }^{-1}\right)\end{array}$ \\
\hline \multicolumn{4}{|c|}{ Horticultural interventions (P) } \\
\hline$P_{0}$ & 213.49 & 47.42 & 75.08 \\
\hline$P_{1}$ & 202.83 & 41.73 & 76.17 \\
\hline$P_{2}$ & 179.58 & 45.90 & 74.72 \\
\hline$P_{3}$ & 191.63 & 46.74 & 82.75 \\
\hline $\mathrm{P}_{4}$ & 165.95 & 39.58 & 77.80 \\
\hline SEm \pm & 1.25 & 0.66 & 0.49 \\
\hline $\operatorname{CD}(p=0.05)$ & 3.53 & 1.86 & 1.46 \\
\hline \multicolumn{4}{|c|}{ Chemical applications (C) } \\
\hline $\mathrm{C}_{0}$ & 192.32 & 39.37 & 73.52 \\
\hline $\mathrm{C}_{1}$ & 189.44 & 45.90 & 78.22 \\
\hline $\mathrm{C}_{2}$ & 188.50 & 48.64 & 81.07 \\
\hline $\mathrm{C}_{3}$ & 192.52 & 43.19 & 76.41 \\
\hline SEm \pm & 1.12 & 0.59 & 0.44 \\
\hline $\mathrm{CD}(p=0.05)$ & 3.16 & 1.66 & 1.34 \\
\hline \multicolumn{4}{|c|}{ Horticultural interventions $\times$ Chemical applications $(\mathrm{P} \times \mathrm{C})$} \\
\hline$P_{0} C_{0}$ & 227.51 & 39.96 & 75.62 \\
\hline $\mathrm{P}_{0} \mathrm{C}_{1}$ & 204.64 & 52.58 & 74.03 \\
\hline $\mathrm{P}_{0} \mathrm{C}_{2}$ & 197.63 & 52.64 & 74.40 \\
\hline $\mathrm{P}_{0} \mathrm{C}_{3}$ & 224.20 & 44.52 & 76.28 \\
\hline $\mathrm{P}_{1} \mathrm{C}_{0}$ & 202.99 & 38.76 & 72.50 \\
\hline $\mathrm{P}_{1} \mathrm{C}_{1}$ & 202.85 & 41.82 & 76.67 \\
\hline $\mathrm{P}_{1} \mathrm{C}_{2}$ & 200.83 & 45.83 & 79.67 \\
\hline $\mathrm{P}_{1} \mathrm{C}_{3}$ & 204.67 & 40.50 & 75.83 \\
\hline $\mathrm{P}_{2} \mathrm{C}_{0}$ & 179.20 & 40.49 & 70.56 \\
\hline $\mathrm{P}_{2} \mathrm{C}_{1}$ & 180.72 & 48.11 & 75.75 \\
\hline $\mathrm{P}_{2} \mathrm{C}_{2}$ & 176.42 & 48.74 & 77.83 \\
\hline$P_{2} C_{3}$ & 181.99 & 46.26 & 74.75 \\
\hline $\mathrm{P}_{3} \mathrm{C}_{0}$ & 189.94 & 40.23 & 76.75 \\
\hline$P_{3} C_{1}$ & 190.78 & 48.55 & 85.00 \\
\hline $\mathrm{P}_{3} \mathrm{C}_{2}$ & 193.39 & 53.86 & 88.33 \\
\hline $\mathrm{P}_{3} \mathrm{C}_{3}$ & 192.42 & 44.33 & 80.92 \\
\hline $\mathrm{P}_{4} \mathrm{C}_{0}$ & 161.98 & 37.41 & 72.19 \\
\hline $\mathrm{P}_{4} \mathrm{C}_{1}$ & 168.21 & 38.46 & 79.63 \\
\hline $\mathrm{P}_{4} \mathrm{C}_{2}$ & 174.25 & 42.14 & 85.11 \\
\hline $\mathrm{P}_{4} \mathrm{C}_{3}$ & 159.35 & 40.32 & 74.25 \\
\hline SEm \pm & 2.51 & 1.32 & 0.97 \\
\hline $\mathrm{CD}(p=0.05)$ & 7.06 & 3.71 & 2.88 \\
\hline
\end{tabular}


$\mathrm{ml} \mathrm{I}^{-1}\left(\mathrm{C}_{2}\right)$ treatment (48.64). The significantly maximum bisexual flowers plant ${ }^{-1}$ (81.07) was registered in ethrel 2 $\mathrm{ml} \mathrm{l}^{-1}\left(\mathrm{C}_{2}\right)$ treatment as compared minimum recorded in control without chemicals $\left(C_{0}\right)$ treatment (73.52). Among interaction treatments, minimum number of male flowers plant $^{-1}$ (159.35) was recorded in withholding irrigation during June+pruning and thinning+ethrel $3 \mathrm{ml} \mathrm{l}^{-1}\left(\mathrm{P}_{4} \mathrm{C}_{3}\right)$ treatment as compared to maximum male flowers plant ${ }^{-1}$ was recorded (227.51) in absolute control $\left(P_{0} C_{0}\right)$ treatment. The maximum number of intermediate flowers plant ${ }^{-1}(53.86)$ was recorded in withholding irrigation during June+ethrel $2 \mathrm{ml} \mathrm{l}^{-1}\left(P_{3} C_{2}\right)$ treatment as compared to absolute control $\left(\mathrm{P}_{0} \mathrm{C}_{0}\right)$ treatment (39.96) treatment. The significantly maximum number of bisexual flowers plant ${ }^{-1}$ (88.33) was recorded in withholding irrigation during June+ethrel $2 \mathrm{ml} \mathrm{l}^{-1}\left(\mathrm{P}_{3} \mathrm{C}_{2}\right)$ treatment as compared to absolute control $\left(\mathrm{P}_{0} \mathrm{C}_{0}\right)$ treatment (75.62). Similarly, Ahire et al. (1993), Chaudhari and Desai (1993), Goswami et al. (2013) and Supe et al. (2015) reported that ethrel application reduced male flowers and increased bisexual flowers in pomegranate. The minimum male flowers in ethrel treatment may be due to the arrest of stamen development by the initiation of DNA damage which promotes female flowers (Wang et al., 2010 and Xie et al., 2015). Pruning alter ratio of old and new growth, crop geometry, sap flow, distribution of photosynthic compounds and encourages more flow of nutrients and water to the remaining shoots which produced more bisexual flowers.

The different interaction treatments affected flowering and harvesting period of pomegranate under hot arid climate (Table 3). In natural flowering without chemicals (absolute control) treatment, flowering were observed during $25^{\text {th }}$ February to $5^{\text {th }}$ April, $21^{\text {st }}$ July to $30^{\text {th }}$ August and $21^{\text {st }}$ September to $12^{\text {th }}$ October while fruits were harvested during $22^{\text {nd }}$ July to $31^{\text {st }}$ August, $3^{\text {rd }}$ December to $13^{\text {th }}$ February and $14^{\text {th }}$ February to $12^{\text {th }}$ March in ambe, mrig and hasta bahar, respectively. Owing to this, there is staggered harvesting and low yield which resulted in poor economics. In withholding irrigation during March $\left(P_{1}\right)$ treatments flowering period was observed $21^{\text {st }}$ May to $8^{\text {th }}$ August while fruits were harvested during $4^{\text {th }}$ November to $10^{\text {th }}$ January among different interactions treatments. In withholding irrigation during March+pruning and thinning $\left(P_{2}\right)$ treatments, flowering period was observed $22^{\text {nd }}$ May to

\begin{tabular}{|c|c|c|c|c|}
\hline $\begin{array}{l}\text { Treat- } \\
\text { ments }\end{array}$ & Flowering period & Harvesting period & Aril colour & Rind colour \\
\hline $\mathrm{P}_{0} \mathrm{C}_{0}$ & $\begin{array}{l}25^{\text {th }} \text { Feb. to } 5^{\text {th }} \text { April } \\
21^{\text {st }} \text { July to } 30^{\text {th }} \text { Aug. \& } 21^{\text {st }} \\
\text { Sep. to } 12^{\text {th }} \text { Oct. }\end{array}$ & $\begin{array}{l}22^{\text {nd }} \text { July to } 31^{\text {st }} \text { Aug. } \\
3^{\text {rd }} \text { Dec. to } 13^{\text {th }} \text { Feb. } \& 14^{\text {th }} \text { Feb. } \\
\text { to } 12^{\text {th }} \text { March }^{*}\end{array}$ & $\begin{array}{l}\text { Orange white } 159 \mathrm{~A}, \\
\text { Red purple } 62 \mathrm{C} \text { and } \\
\text { Orange white } 159 \mathrm{~B}^{*}\end{array}$ & $\begin{array}{l}\text { Grayed orange } 163 \mathrm{~A} \text {, } \\
\text { Grayed orange } 163 \mathrm{C} \text { and } \\
\text { Grayed orange } 163 C^{*}\end{array}$ \\
\hline $\mathrm{P}_{0} \mathrm{C}_{1}$ & $1^{\text {st }}$ July to $16^{\text {th }}$ Sep. & $13^{\text {th }}$ Dec. to $16^{\text {th }}$ Feb. & Red purple 62A & Grayed orange $163 C$ \\
\hline $\mathrm{P}_{0} \mathrm{C}_{2}$ & $5^{\text {th }}$ July to $18^{\text {th }}$ Sep. & $19^{\text {th }}$ Dec. to $18^{\text {th }}$ Feb. & Red purple 63B & Grayed orange 163B \\
\hline $\mathrm{P}_{0} \mathrm{C}_{3}$ & $9^{\text {th }}$ July to $9^{\text {th }}$ Sep. & $25^{\text {th }}$ Dec. to $20^{\text {th }}$ Feb. & Red purple 62C & Grayed orange 163B \\
\hline $\mathrm{P}_{1} \mathrm{C}_{0}$ & $24^{\text {th }}$ May to $8^{\text {th }}$ Aug. & $4^{\text {th }}$ Nov. to $3^{\text {rd }}$ Jan. & Red purple 62A & Green yellow 1B \\
\hline $\mathrm{P}_{1} \mathrm{C}_{1}$ & $21^{\text {st }}$ May to $4^{\text {th }}$ Aug & $5^{\text {th }}$ Nov. to $6^{\text {th }}$ Jan. & Red purple 63A & Grayed orange $163 C$ \\
\hline $\mathrm{P}_{1} \mathrm{C}_{2}$ & $27^{\text {th }}$ May to $8^{\text {th }}$ Aug. & $14^{\text {th }}$ Nov. to $7^{\text {th }}$ Jan. & Red purple 63A & Grayed orange 163B \\
\hline $\mathrm{P}_{1} \mathrm{C}_{3}$ & $29^{\text {th }}$ May to $28^{\text {th }}$ July & $18^{\text {th }}$ Nov. to $10^{\text {th }}$ Jan. & Red purple 63A & Grayed orange 163A \\
\hline $\mathrm{P}_{2} \mathrm{C}_{0}$ & $27^{\text {th }}$ May to $6^{\text {th }}$ Aug. & $6^{\text {th }}$ Nov. to $6^{\text {th }}$ Jan. & Red purple 62C & Green yellow $1 \mathrm{~B}$ \\
\hline $\mathrm{P}_{2} \mathrm{C}_{1}$ & $22^{\text {nd }}$ May to $30^{\text {th }}$ July & $8^{\text {th }}$ Nov. to $7^{\text {th }}$ Jan. & Red purple 62A & Grayed orange $163 C$ \\
\hline $\mathrm{P}_{2} \mathrm{C}_{2}$ & $31^{\text {st }}$ May to $7^{\text {th }}$ Aug. & $14^{\text {th }}$ Nov. to $11^{\text {th }}$ Jan. & Red purple 63B & Grayed orange 163B \\
\hline $\mathrm{P}_{2} \mathrm{C}_{3}$ & $3^{\text {rd }}$ June to $10^{\text {th }}$ Aug. & $19^{\text {th }}$ Nov. to $15^{\text {th }}$ Jan. & Red purple 62A & Grayed orange $163 \mathrm{~A}$ \\
\hline $\mathrm{P}_{3} \mathrm{C}_{0}$ & $21^{\text {st }}$ Aug. to $2^{\text {nd }}$ Nov. & $11^{\text {th }}$ Jan. to $9^{\text {th }}$ March & Red purple 62C & Grayed orange $163 C$ \\
\hline $\mathrm{P}_{3} \mathrm{C}_{1}$ & $31^{\text {st }}$ July to $6^{\text {th }}$ Oct. & $13^{\text {th }}$ Jan. to $11^{\text {th }}$ March & Red purple 62B & Grayed orange N-163D \\
\hline $\mathrm{P}_{3} \mathrm{C}_{2}$ & $3^{\text {rd }}$ Aug. to $7^{\text {th }}$ Oct. & $17^{\text {th }}$ Jan. to $15^{\text {th }}$ March & Red purple 62A & Grayed orange N-163C \\
\hline $\mathrm{P}_{3} \mathrm{C}_{3}$ & $6^{\text {th }}$ Aug. to 4th Oct. & $21^{\text {st }}$ Jan. to $9^{\text {th }}$ March & Red purple 62C & Grayed orange N-163B \\
\hline $\mathrm{P}_{4} \mathrm{C}_{0}$ & $25^{\text {th }}$ Aug. to $5^{\text {th }}$ Nov. & $13^{\text {th }}$ Jan. to $12^{\text {th }}$ March & Red purple 62C & Grayed orange $163 C$ \\
\hline $\mathrm{P}_{4} \mathrm{C}_{1}$ & $1^{\text {st }}$ Aug. to $6^{\text {th }}$ Oct. & $17^{\text {th }}$ Jan. to $16^{\text {th }}$ March & Red purple 62C & Grayed orange N-163D \\
\hline $\mathrm{P}_{4} \mathrm{C}_{2}$ & $8^{\text {th }}$ Aug. to $12^{\text {th }}$ Oct. & $19^{\text {th }}$ Jan. to $20^{\text {th }}$ March & Red purple 62B & Grayed orange N-163C \\
\hline $\mathrm{P}_{4} \mathrm{C}_{3}$ & $15^{\text {th }}$ Aug. to $10^{\text {th }}$ Oct. & $23^{\text {rd }}$ Jan. to $28^{\text {th }}$ March & Red purple $62 \mathrm{C}$ & Grayed orange N-163B \\
\hline
\end{tabular}

*ambe, mrig and hasta bahar, respectively 
$10^{\text {th }}$ August among different interactions treatments while fruits were harvested during $6^{\text {th }}$ November to $15^{\text {th }}$ January. In withholding irrigation during June $\left(P_{3}\right)$ treatments, flowering period was observed $3^{\text {rd }}$ August to $7^{\text {th }}$ October while fruits were harvested during $11^{\text {th }}$ January to $15^{\text {th }}$ March among different interactions treatments. In withholding irrigation during June+pruning and thinning $\left(\mathrm{P}_{4}\right)$ treatments flowering period was observed $1^{\text {st }}$ August to $12^{\text {th }}$ October while fruits were harvested during $13^{\text {th }}$ January to $28^{\text {th }}$ March among different interactions treatments.

The perusal of data (Table 3 ) showed that horticultural interventions, chemical treatments and their interaction affected aril and rind colour of pomegranate fruits. In absolute control i.e. natural flowering without chemicals $\left(\mathrm{P}_{0} \mathrm{C}_{0}\right)$ treatment aril colour was varied from orange white 159A in ambe bahar, red purple 62C in mrig bahar and orange white 159B in hasta bahar whereas in all other treatment aril colour was varied from red purple 62A, red purple 62B, red purple $62 \mathrm{C}$, red purple $63 \mathrm{~A}$ to red purple $63 \mathrm{~B}$ among different treatment combinations. Dark colour of arils i.e. red purple 63A was observed in withholding irrigation during March+ethrel $1 \mathrm{ml} \mathrm{I}^{-1}\left(\mathrm{P}_{1} \mathrm{C}_{1}\right)$ treatment, withholding irrigation during March+ethrel $2 \mathrm{ml} \mathrm{l}^{-1}\left(\mathrm{P}_{1} \mathrm{C}_{2}\right)$ treatment and withholding irrigation during March+ethrel $3 \mathrm{ml} \mathrm{l}^{-1}\left(\mathrm{P}_{1} \mathrm{C}_{3}\right)$ treatment while medium dark colour i.e red purple 63B was observed in natural flowering+ethrel $2 \mathrm{ml} \mathrm{l}^{-1}\left(\mathrm{P}_{0} \mathrm{C}_{2}\right)$ treatment and withholding irrigation during March+pruning and thinning+ethrel $2 \mathrm{ml}$ $\mathrm{I}^{-1}\left(\mathrm{P}_{2} \mathrm{C}_{2}\right)$ treatment. Similarly in natural flowering without chemicals $\left(P_{0} C_{0}\right)$ treatment rind colour was varied from grayed orange 163A in ambe bahar, grayed orange $163 \mathrm{C}$ in both mrig bahar and hasta bahar whereas in all other treatment rind colour was varied from grayed orange 163A, grayed orange $163 \mathrm{~B}$, grayed orange $163 \mathrm{C}$, grayed orange $\mathrm{N}-163 \mathrm{~B}$, grayed orange $\mathrm{N}-163 \mathrm{C}$, grayed orange $\mathrm{N}-163 \mathrm{D}$ to green yellow $1 \mathrm{~B}$ among different treatment combinations. Dark grayed orange colour of rind i.e. grayed orange N-163B was observed in withholding irrigation during June+ethrel $3 \mathrm{ml} \mathrm{l}^{-1}\left(\mathrm{P}_{3} \mathrm{C}_{3}\right)$ treatment and in withholding irrigation during June+pruning and thinning+ethrel $3 \mathrm{mll}^{-1}\left(\mathrm{P}_{4} \mathrm{C}_{3}\right)$ treatment. The variation in aril and rind might be due to seasonal variation along with pruning which influenced light interception. The increased light interception stimulated exterior fruit colour and improved visual appearance of the fruits. Prasad et al. (1997) reported that due to hot weather during July-August and inferior colour development during January-February, both hasta and ambe bahar was not recommended in arid and semi-arid climate in pomegranate. Ghosh et al. (2012) found that colour of aril was changed with time of maturity. It was pink when the fruits were harvested during May and became light red in June and red in July. Ranpise et al. (2014) found that in pomegranate arils anthocyanin content was generally lower in fruit harvested during June-July and higher in the fruit harvested during November-December. The decrease in anthocyanin content was possibly due to degradation of anthocyanins by high sunlight intensity observed during May-June.

The data showed that horticultural interventions, chemical treatments and their interaction significantly influenced fruit volume. The horticultural interventions and chemical treatments significantly influenced juice content while their interaction have non-significant effect (Table 4). Among different horticultural interventions, significantly maximum fruit volume (255.91 cc) and juice content $(77.56 \%)$ were recorded in withholding irrigation during June+pruning and thinning $\left(P_{4}\right)$ treatment as against minimum fruit volume (207.36 cc) and juice content $(65.87 \%)$ were recorded in control natural flowering $\left(P_{0}\right)$ treatment. Among different chemical applications, maximum fruit volume (242.06 cc) and juice content (73.09\%) were recorded in ethrel $2 \mathrm{ml} \mathrm{l}^{-1}$ $\left(\mathrm{C}_{2}\right)$ treatment as compared to minimum fruit volume $(227.48$ cc) and juice content (70.45\%) recorded in control without chemicals $\left(C_{0}\right)$ treatment. Among interaction treatments, significantly maximum fruit volume $(264.80 \mathrm{cc}$ ) and juice content (78.96\%) were recorded in withholding irrigation during June+pruning and thinning+ethrel $2 \mathrm{ml} \mathrm{l}^{-1}\left(\mathrm{P}_{4} \mathrm{C}_{2}\right)$ treatment whereas minimum fruit volume (201.46 cc) and juice content (65.25\%) were recorded in absolute control $\left(P_{0} C_{0}\right)$ treatment. The higher fruit volume and juice content in withholding irrigation during June+pruning and thinning $\left(\mathrm{P}_{4}\right)$ treatment is possibly due to favourable growth condition and diversion of more nutrients and water to growing fruits by pruning and higher photosynthetic efficiency. These results are in accordance with Sheikh and Rao (2002). Similarly, Goswami et al. (2013) and Supe et al. (2015) reported that ethrel application increased fruit volume in pomegranate. Sharma and Singh (2000) reported that juice percentage was more due to deblossoming in April month as compared to control in pomegranate. The translocation of sugars and water to the arils tends to be the cause of the rise in juice volume. The proportion of seeds may have decreased in proportion to the increase in the amount of juice as the water content of arils increased (Supe and Saitwal, 2016).

The data revealed that horticultural interventions, chemical treatments and their interaction significantly improved maturity index of pomegranate. Among horticultural interventions, significantly maximum maturity index (45.18) was recorded in withholding irrigation during June+pruning and thinning $\left(P_{4}\right)$ treatment as compared to control $\left(P_{0}\right)$ with maturity index (35.90). Among chemical treatments, significantly maximum maturity index (38.65) was registered in ethrel $2 \mathrm{ml} \mathrm{l}^{-1}\left(C_{2}\right)$ treatment in comparison to minimum maturity index (33.99) recorded in control without chemicals $\left(C_{0}\right)$ treatment. Among interaction treatments, significantly maximum maturity index (47.81) was recorded in withholding irrigation during June+pruning and thinning+ethrel $2 \mathrm{ml} \mathrm{l}^{-1}$ $\left(\mathrm{P}_{4} \mathrm{C}_{2}\right)$ treatment as compared minimum maturity index (27.01) recorded in withholding irrigation during March without chemicals $\left(\mathrm{P}_{1} \mathrm{C}_{0}\right)$ treatment. The maturity is mainly related 
Table 4: Effect of flower regulation treatments on fruit quality and yield attributes of pomegranate

\begin{tabular}{lcccc}
\hline Treatments & $\begin{array}{c}\text { Fruit volume } \\
\text { (cc) }\end{array}$ & $\begin{array}{c}\text { Juice } \\
(\%)\end{array}$ & $\begin{array}{c}\text { Maturity } \\
\text { index }\end{array}$ & $\begin{array}{c}\text { Fruit yield } \\
\left(\mathrm{q} \mathrm{ha} \mathrm{h}^{-1}\right)\end{array}$ \\
\hline \multicolumn{4}{l}{ Horticultural interventions $(\mathrm{P})$} \\
\hline $\mathrm{P}_{0}$ & 207.36 & 65.87 & 35.90 & 93.52 \\
$\mathrm{P}_{1}$ & 227.29 & 68.92 & 28.65 & 100.11 \\
$\mathrm{P}_{2}$ & 244.39 & 71.40 & 30.86 & 101.40 \\
$\mathrm{P}_{3}$ & 235.51 & 74.18 & 41.32 & 106.65 \\
$\mathrm{P}_{4}$ & 255.91 & 77.56 & 45.18 & 105.98 \\
$\mathrm{SEm} \pm$ & 0.63 & 0.35 & 0.15 & 0.85 \\
$\mathrm{CD}(p=0.05)$ & 1.77 & 0.99 & 0.42 & 2.40
\end{tabular}

Chemical applications (C)

\begin{tabular}{lccccc}
\cline { 1 - 2 }$C_{0}$ & 227.48 & & 70.45 & 33.99 & 92.16 \\
$C_{1}$ & 236.19 & & 71.73 & 37.27 & 104.42 \\
$C_{2}$ & 242.06 & 73.09 & 38.65 & 111.47 \\
$C_{3}$ & 230.64 & & 71.08 & 35.61 & 98.09 \\
SEm \pm & 0.56 & 0.31 & 0.13 & 0.76 \\
$C D(p=0.05)$ & 1.58 & 0.88 & 0.37 & 2.15
\end{tabular}

Horticultural interventions $\times$ Chemical applications $(\mathrm{P} \times \mathrm{C})$

\begin{tabular}{lcccc}
\hline $\mathrm{P}_{0} \mathrm{C}_{0}$ & 201.46 & 65.25 & 33.35 & 85.66 \\
$\mathrm{P}_{0} \mathrm{C}_{1}$ & 210.12 & 65.82 & 36.93 & 95.68 \\
$\mathrm{P}_{0} \mathrm{C}_{2}$ & 211.75 & 67.54 & 37.95 & 99.42 \\
$\mathrm{P}_{0} \mathrm{C}_{3}$ & 206.10 & 64.89 & 35.37 & 93.31 \\
$\mathrm{P}_{1} \mathrm{C}_{0}$ & 218.59 & 67.74 & 27.01 & 92.35 \\
$\mathrm{P}_{1} \mathrm{C}_{1}$ & 231.68 & 69.27 & 29.13 & 103.09 \\
$\mathrm{P}_{1} \mathrm{C}_{2}$ & 237.10 & 70.25 & 30.31 & 107.14 \\
$\mathrm{P}_{1} \mathrm{C}_{3}$ & 221.80 & 68.42 & 28.14 & 97.88 \\
$\mathrm{P}_{2} \mathrm{C}_{0}$ & 238.56 & 70.01 & 29.42 & 92.25 \\
$\mathrm{P}_{2} \mathrm{C}_{1}$ & 243.72 & 71.73 & 31.17 & 105.82 \\
$\mathrm{P}_{2} \mathrm{C}_{2}$ & 254.00 & 72.76 & 32.70 & 108.91 \\
$\mathrm{P}_{2} \mathrm{C}_{3}$ & 241.30 & 71.11 & 30.13 & 98.62 \\
$\mathrm{P}_{3} \mathrm{C}_{0}$ & 229.18 & 72.50 & 37.69 & 97.06 \\
$\mathrm{P}_{3} \mathrm{C}_{1}$ & 238.07 & 74.17 & 42.38 & 108.78 \\
$\mathrm{P}_{3} \mathrm{C}_{2}$ & 242.65 & 75.93 & 44.49 & 117.87 \\
$\mathrm{P}_{3} \mathrm{C}_{3}$ & 232.14 & 74.10 & 40.74 & 102.88 \\
$\mathrm{P}_{4} \mathrm{C}_{0}$ & 249.62 & 76.74 & 42.47 & 93.47 \\
$\mathrm{P}_{4} \mathrm{C}_{1}$ & 257.38 & 77.66 & 46.75 & 108.71 \\
$\mathrm{P}_{4} \mathrm{C}_{2}$ & 264.80 & 78.96 & 47.81 & 124.01 \\
$\mathrm{P}_{4} \mathrm{C}_{3}$ & 251.83 & 76.88 & 43.69 & 97.75 \\
$\mathrm{SEm} \pm$ & 1.26 & 0.70 & 0.30 & 1.71 \\
$\mathrm{CD}(p=0.05)$ & 3.54 & $\mathrm{NS}$ & 0.83 & 4.80 \\
\hline & & & &
\end{tabular}

to palatability of fruit than either sugar or acid level alone. It varies from 31.90 to 37.90 among different cultivars (Dhinesh et al., 2017). The higher maturity index in withholding irrigation during June+pruning and thinning+ethrel $2 \mathrm{ml} \mathrm{l}^{-1}$ $\left(\mathrm{P}_{4} \mathrm{C}_{2}\right)$ treatment probably due to higher TSS and lower acidity because of optimal climatic condition, more flow of nutrients and assimilates to growing fruits by pruning and thinning effect. In ethrel application, lower acidity may be owing to increased respiration and carbon assimilation activities which resulted in conversion of complex carbohydrates (starch) to simple carbohydrates (sugar). Ethrel induced stress may also increased water soluble osmolytes which decreased acidity. Sheikh and Rao (2002) observed that fruit TSS significantly influenced with pruning intensity and maximum TSS was recorded in higher pruning intensity.

The horticultural interventions, chemical treatments and their interaction significantly improved fruit yield in pomegranate. Among horticultural interventions, significantly maximum fruit yield (106.65 q ha-1) was recorded in with holding irrigation during June $\left(\mathrm{P}_{3}\right)$ treatment. The minimum fruit yield $(93.52 \mathrm{q}$ ha $^{-1}$ ) was recorded in control natural flowering $\left(P_{0}\right)$ treatment. Among chemical applications, maximum fruit yield (111.47 q $\mathrm{ha}^{-1}$ ) was registered in ethrel $2 \mathrm{mll}^{-1}\left(\mathrm{C}_{2}\right)$ treatment as against minimum recorded in control without chemicals $\left(C_{0}\right)$ treatment $\left(92.16 \mathrm{q} \mathrm{ha}^{-1}\right)$. Among interaction treatments, maximum fruit yield was recorded (124.01 $\mathrm{q} \mathrm{ha}^{-1}$ ) in withholding irrigation during June+pruning and thinning+ethrel $2 \mathrm{ml} \mathrm{l}^{-1}\left(\mathrm{P}_{4} \mathrm{C}_{2}\right)$ treatment which was followed by withholding irrigation during June+ethrel $2 \mathrm{mll}^{-1}\left(\mathrm{P}_{3} \mathrm{C}_{2}\right)$ treatment $\left(117.87 \mathrm{q} \mathrm{ha}^{-1}\right)$. The minimum fruit yield $\left(85.66 \mathrm{q} \mathrm{ha}^{-1}\right)$ was recorded in absolute control $\left(P_{0} C_{0}\right)$ treatment. The higher yield in withholding irrigation during June+pruning and thinning+ethrel $2 \mathrm{ml} \mathrm{l}^{-1}$ $\left(\mathrm{P}_{4} \mathrm{C}_{2}\right)$ treatments might be due to favourable growth condition which resulted higher fruit set and size while application of ethrel increased number of bisexual flowers with high fruit set and retention. These findings are in agreement with Sheikh and Rao (2002) who observed that fruit yield was significantly influenced by pruning intensity. Similar results were also noted by Goswami et al. (2013) who reported that ethrel application improved fruit yield in pomegranate as compared to minimum recorded in control. Supe et al. (2015) reported that spraying of ethrel $2 \mathrm{ml} \mathrm{l}^{-1}$ mixed with DAP $5 \mathrm{~g}^{-1}$ increased fruit yield in pomegranate.

The data revealed that horticultural interventions, chemical treatments and their interaction significantly reduced fruit splitting, mite and fungal spot incidence in pomegranate (Table 5). Among horticultural interventions, significantly minimum fruit splitting (1.66 $\left.\mathrm{kg} \mathrm{plant}^{-1}\right)$ was recorded in withholding irrigation during June+pruning and thinning $\left(\mathrm{P}_{4}\right)$ treatment as compared to control $\left(\mathrm{P}_{0}\right)$ with fruit splitting ( 2.54 $\mathrm{kg}$ plant $\left.{ }^{-1}\right)$. Among chemical treatments, significantly minimum fruit splitting (2.04 kg plant ${ }^{-1}$ ) was registered in ethrel $2 \mathrm{ml}$ $\mathrm{I}^{-1}\left(\mathrm{C}_{2}\right)$ treatment in comparison to maximum fruit splitting $\left(2.37 \mathrm{~kg} \mathrm{plant}^{-1}\right)$ recorded in control without chemicals $\left(C_{0}\right)$ 


\begin{tabular}{|c|c|c|c|}
\hline Treatments & $\begin{array}{c}\text { Fruit splitting } \\
\left(\text { kg plant }^{-1}\right)\end{array}$ & $\begin{array}{c}\text { Mite } \\
\text { incidence (\%) }\end{array}$ & $\begin{array}{c}\text { Fungal spot } \\
\text { incidence (\%) }\end{array}$ \\
\hline \multicolumn{4}{|c|}{ Horticultural interventions (P) } \\
\hline$P_{0}$ & 2.54 & 20.33 & 21.35 \\
\hline$P_{1}$ & 2.58 & 17.19 & 17.94 \\
\hline$P_{2}$ & 2.17 & 14.10 & 16.94 \\
\hline$P_{3}$ & 2.33 & 11.64 & 14.60 \\
\hline $\mathrm{P}_{4}$ & 1.66 & 9.63 & 12.72 \\
\hline SEm \pm & 0.04 & 0.47 & 0.54 \\
\hline $\mathrm{CD}(p=0.05)$ & 0.13 & 1.31 & 1.53 \\
\hline \multicolumn{4}{|c|}{ Chemical applications (C) } \\
\hline $\mathrm{C}_{0}$ & 2.37 & 23.70 & 23.87 \\
\hline $\mathrm{C}_{1}$ & 2.27 & 17.11 & 19.92 \\
\hline $\mathrm{C}_{2}$ & 2.04 & 10.01 & 12.71 \\
\hline $\mathrm{C}_{3}$ & 2.35 & 7.50 & 10.34 \\
\hline SEm \pm & 0.04 & 0.42 & 0.49 \\
\hline $\mathrm{CD}(p=0.05)$ & 0.11 & 1.17 & 1.37 \\
\hline \multicolumn{4}{|c|}{ Horticultural interventions $\times$ Chemical applications $(\mathrm{P} \times \mathrm{C})$} \\
\hline $\mathrm{P}_{0} \mathrm{C}_{0}$ & 2.41 & 29.73 & 33.68 \\
\hline$P_{0} C_{1}$ & 2.68 & 18.61 & 24.99 \\
\hline $\mathrm{P}_{0} \mathrm{C}_{2}$ & 2.26 & 18.82 & 14.06 \\
\hline $\mathrm{P}_{0} \mathrm{C}_{3}$ & 2.80 & 14.16 & 12.67 \\
\hline$P_{1} C_{0}$ & 2.87 & 29.27 & 25.33 \\
\hline$P_{1} C_{1}$ & 2.52 & 20.31 & 22.71 \\
\hline$P_{1} C_{2}$ & 2.20 & 12.43 & 13.58 \\
\hline$P_{1} C_{3}$ & 2.74 & 6.78 & 10.14 \\
\hline $\mathrm{P}_{2} \mathrm{C}_{0}$ & 2.38 & 24.39 & 24.20 \\
\hline $\mathrm{P}_{2} \mathrm{C}_{1}$ & 2.14 & 19.11 & 19.89 \\
\hline $\mathrm{P}_{2} \mathrm{C}_{2}$ & 1.82 & 6.93 & 13.12 \\
\hline$P_{2} C_{3}$ & 2.34 & 5.98 & 10.54 \\
\hline $\mathrm{P}_{3} \mathrm{C}_{0}$ & 2.50 & 18.23 & 19.03 \\
\hline $\mathrm{P}_{3} \mathrm{C}_{1}$ & 2.44 & 16.33 & 16.69 \\
\hline $\mathrm{P}_{3} \mathrm{C}_{2}$ & 2.03 & 6.13 & 12.81 \\
\hline$P_{3} C_{3}$ & 2.33 & 5.86 & 9.87 \\
\hline $\mathrm{P}_{4} \mathrm{C}_{0}$ & 1.67 & 16.87 & 17.11 \\
\hline $\mathrm{P}_{4} \mathrm{C}_{1}$ & 1.54 & 11.17 & 15.31 \\
\hline $\mathrm{P}_{4} \mathrm{C}_{2}$ & 1.87 & 5.75 & 9.97 \\
\hline $\mathrm{P}_{4} \mathrm{C}_{3}$ & 1.55 & 4.72 & 8.50 \\
\hline SEm \pm & 0.09 & 0.93 & 1.09 \\
\hline $\mathrm{CD}(p=0.05)$ & 0.25 & 2.62 & 3.07 \\
\hline
\end{tabular}

treatment. Among interaction treatments, significantly lower fruit splitting (1.87 kg plant $\left.{ }^{-1}\right)$ was recorded in withholding irrigation during June+pruning and thinning+ethrel $2 \mathrm{ml} \mathrm{l}^{-1}$ $\left(\mathrm{P}_{4} \mathrm{C}_{2}\right)$ treatment as compared maximum fruit splitting $(2.87$ $\mathrm{kg} \mathrm{plant}^{-1}$ ) were recorded in withholding irrigation during March without chemicals $\left(P_{1} C_{0}\right)$ treatment. The low fruit splitting in withholding irrigation during June+pruning and thinning+ethrel $2 \mathrm{mll}^{-1}\left(\mathrm{P}_{4} \mathrm{C}_{2}\right)$ treatment might be attributed to induction of flowering and fruit development during favorable climatic condition, low variation in diurnal temperature, low incidence of mite and fungal spot under hot arid climate.

Among different horticultural interventions, significantly minimum mite $(9.63 \%)$ and fungal spot $(12.72 \%)$ incidence were recorded in withholding irrigation during June+pruning and thinning $\left(\mathrm{P}_{4}\right)$ treatment as compared to maximum mite $(20.33 \%)$ and fungal spot $(21.35 \%)$ incidence recorded in control natural flowering $\left(\mathrm{P}_{0}\right)$ treatment. Among chemical treatments, minimum mite $(7.50 \%)$ and fungal spot $(10.34 \%)$ incidence were recorded in ethrel $3 \mathrm{ml} \mathrm{l}^{-1}\left(C_{3}\right)$ treatment as against maximum mite $(23.70 \%)$ and fungal spot $(23.87 \%)$ incidence were recorded in control without chemicals $\left(C_{0}\right)$ treatment. Among different combinations of horticultural interventions and chemical treatments, minimum mite $(4.72 \%)$ and fungal spot $(8.50 \%)$ incidence were recorded in withholding irrigation during June+pruning and thinning+ethrel $3 \mathrm{ml} \mathrm{l}^{-1}\left(\mathrm{P}_{4} \mathrm{C}_{3}\right)$ treatment as compared to maximum mite $(29.73 \%)$ and fungal spot $(33.68 \%)$ incidence recorded in absolute control $\left(\mathrm{P}_{0} \mathrm{C}_{0}\right)$ treatment. The lower incidence of mite and fungal spot in withholding irrigation during June+pruning and thinning+ethrel $3 \mathrm{ml} \mathrm{l}^{-1}\left(\mathrm{P}_{4} \mathrm{C}_{3}\right)$ treatment was probably due to defoliation of leaves caused by water and chemical (ethrel) stress which minimized pest population and disease inoculums and induced new foliage free from pest and disease. Pruning and thinning caused proper ventilation and improved plant health owing to more flow of nutrients, water and photosynthetic assimilates. Application of ethylene $0.2 \mathrm{ml} \mathrm{l}^{-1}$ reduced bacterial blight incidence and enhanced fruit yield/quality in pomegranate (Lalithya et al., 2017). Sharma and Singh (2018) also observed that increasing pruning intensity with thinning significantly reduced disease severity on fruit and leaf surface of pomegranate.

\section{Conclusion}

In pomegranate crop regulation, water stress, ethrel application and bahar pruning were influenced flowering induction, altered sex ratio and improved quality fruit yield. Water stress induced flowering during optimal climatic period which resulted in reduction of fruit splitting with improvement in fruit quality and yield. Bahar pruning balanced vegetative and reproductive growth which regulated flowering and fruiting for quality yield. Crop regulation also reduced mite and fungal spot incidence in pomegranate through defoliation and enhanced management. 


\section{Acknowledgement}

The authors are grateful to the ICAR-Central Institute for Arid Horticulture, Bikaner for providing required facilities and guidance to conduct the research.

\section{References}

Abeles, F.B., Morgan, P.W., Saltveit Jr, M.E., 1992. Ethylene in plant biology. $2^{\text {nd }}$ ed. Academic Press, San Diego.

Ahire, G.Z., Desai, U.T., Choudhari, S.M., Masalkar, S.D., 1993. Crop regulation in pomegranate: I. Effect of growth regulators on flower induction, sex expression and flower drop. Annals Arid Zone 32, 97-98.

Amarnath, K.S., Mishra, S., Singh, R.K., 2020. Effect of pruning in pomegranate (Punica granatum L.) for shoot growth, flowering and fruit yield. Current Journal of Applied Science and Technology 39(30), 114-123.

Anonymous, 2021. Area and production of horticulture crops for 2020-21 ( $3^{\text {rd }}$ Advance Estimate). Department of Agriculture, Cooperation \& Farmers' Welfare, Government of India, 1.

Chaudhari, S.M., Desai, U.T., 1993. Effects of plant growth regulators on flower sex in pomegranate (Punica granatum L.). Indian Journal of Agricultural Sciences 63, 34-35.

Dhinesh, B.K., Singh, N.V., Gaikwad, N., Maity, A., Suryavanshi, S.K., Pal, R.K., 2017. Determination of maturity indices for harvesting of pomegranate (Punica granatum). Indian Journal of Agricultural Sciences 87(9), 1225-1230.

Galindo, A., Rodriguez, P., Collado-Gonzalez, J., Cruz, Z.N., Torrecillas, E., Ondono, S., Corell, M., Moriana, A., Torrecillas, A., 2014. Rainfall intensifies fruit peel cracking in water stressed pomegranate trees. Agricultural and Forest Meteorology 194, 29-35

Ghosh, S.N., Bera, B.R., Kundu, S.A., Bhattacharyya, A., 2012. Effect of crop management factors on pomegranate cultivation in West Bengal. Acta Horticulturae 940, 163-170.

Goswami, J.D., Patel, N.M., Bhadauria, H.S., Wankhade, V.R., 2013. Effect of plant growth substances on growth, fruit setting and yield of pomegranate cv. Sinduri. International Journal of Agricultural Sciences 9(1), 32-34.

Kumar, R., Berwal, M.K., Saroj, P.L., 2019a. Morphological, physiological, biochemical and molecular facet of drought stress in horticultural crops. International Journal of Bio-resource and Stress Management 10(5), 545-560.

Kumar, R., Saroj, P.L., Sharma, B.D., 2019b. Flower regulation in pomegranate for higher yield, improved quality and enhanced management-a review. Fruits, The International Journal of Tropical and Subtropical Horticulture 74(4), 150-166.

Kumar, R., Saroj, P.L., Sharma, B.D., Yadav, P.K., 2020. Studies on flowering induction, sex ratio and fruit set improvement in pomegranate. Indian Journal of Horticulture 77(4), 610-618.

Lalithya, K.A., Manjunatha, G., Raju, B., Kulkarni, M.S., Lokesh, V., 2017. Plant growth regulators and signal molecules enhance resistance against bacterial blight disease of pomegranate. Phytopathology 165, 727-736.

Panse, V.G., Sukhatme, P.V., 1985. Statistical methods for agricultural workers. Fourth Enlarged Edition, ICAR publication, New Delhi.

Prasad, R.N., Bankar, G.J., Vashishtha, B.B., 1997. Problems and prospects of pomegranate cultivation in arid region. In:Proceedings of symposium on recent advances in management of arid ecosystem, Jodhpur, Rajasthan.

Rajkumar, Gora, J.S., Kumar, R., Singh, A., Kumar, A., Gajendera, 2017. Effect of different growing media on the rooting of pomegranate (Punica granatum L.) cv. 'Phule Arakta' cuttings. Journal of Applied and Natural Science 9(2), 715-719.

Ranganna, S., 1995. Handbook of analysis and quality control for fruits and vegetable products. McGraw Hill Publishing Company Limited, New Delhi.

Ranpise, S.A., Sankaran, M., Babu, K.D., Prakash, J., Hiwale, S.S., 2014. Pomegranate. In: Gosh, S.N. (Ed.), Tropical and sub tropical fruit crops- Crop improvement and varietal wealth. , Jaya Publishing House, Delhi, 549-573.

Sachin, A.J., Ramteke, V., Bharath, K.R., 2015. Flower regulation in guava and pomegranate. Popular Kheti 3, 53-54.

Saroj, P.L., Kumar, R., 2019. Recent advances in pomegranate production in India-a review. Annals of Horticulture 12(1), 1-10.

Sharma, D.P., Singh, N., 2018. Effect of rejuvenation pruning on the growth, productivity and disease incidence in declining trees of pomegranate (Punica granatum L.) cv. Kandhari Kabuli. Journal of Applied and Natural Science 10(1), 358-362.

Sharma, K.K., Singh, N.P., 2000. Effect of mechanical deblossoming on yield and quality of pomegranate (Punica granatum L.). Journal of Research, Punjab Agricultural University, 37, 203-205.

Sheikh, M.K., Rao, M.M., 2002. Effect of pruning and fruit load on yield and quality in pomegranate var. Ganesh. Karnataka Journal of Agricultural Sciences 15, 549-555.

Shivran, J.S., Jat, M.L., Jat, R.K., 2020. Crop regulation in pomegranate. Journal of Crop and Weed 16(1), 242-244.

Singh, D.B., Kingsly, A.R., 2007. Regulation of bahar in pomegranate (Punica granatum) to control fruit cracking and improve the quality. Indian Journal of Agricultural Sciences 77(10), 692-94.

Supe, V.S., Joshi, V.R., Patil, S.D., Attar, A.V., 2015. Effect of different chemicals on leaf shedding of pomegranate 'Bhagwa'. In: Yuan, Z., Wilkins, E., Wang, D. (Eds.), Proc. III ${ }^{\text {rd }}$ IS on Pomegranate and Minor Mediterranean Fruits, Acta Horticulturae 1089, 437-442.

Supe, V.S., Saitwal, Y.S., 2016. Morphological, biochemical 
and qualitative changes associated with growth and development of pomegranate fruit (Punica granatum L.). Indian Journal of Agricultural Research 50, 80-83.

Wang, D.H., Li, F., Duan, Q.H., Han, T., Xu, Z.H., Bai, S.N., 2010. Ethylene perception is involved in female cucumber flower development. Plant Journal 61, 862-872.

Westwood, M.N., 1978. Plant efficiency: growth and yield measurement. Temperate zone pomology. W.H. Freeman and Co., San Franscisco, U.S.A. 220-228.

Wetzstein, H.Y., Porter, J.A., Ravid, N., 2015. Reproductive biology of pomegranate from flowering to fruit development. Acta Horticulturae 1089, 21-28.

Xie, S., Lu, X., Zhao, X., Nie, Q., 2015. Effect of water stress on vegetative growth and nitrogen metabolism of pomegranate seedling. Acta Horticulturae 1089, 63-69. Yahya, S., Zabihollah, Z., Reza, F., Ali-Reza, T., 2017. Effect of deficit irrigation on flowering and fruit properties of pomegranate (Punica granatum cv. Shahvar). Agricultural Water Management 192, 189-197. 